\title{
Tuberculosis Arthritis of the Sternoclavicular Joint
}

\author{
Osman Walid1, Triki Mohamed Amine1, Kaziz Hamdi', Jemni Sonia², Naouar Nader1, \\ Ben Ayech Mohamed Laziz ${ }^{1}$ \\ ${ }^{1}$ Department of Orthopedic Surgery, University Hospital Sahloul, Sousse, Tunisia \\ ${ }^{2}$ Physiotherapy Department, University Hospital Sahloul, Sousse, Tunisia \\ Email: Trikos@hotmail.fr
}

Received 4 May 2015; accepted 8 June 2015; published 11 June 2015

Copyright (C) 2015 by authors and Scientific Research Publishing Inc.

This work is licensed under the Creative Commons Attribution International License (CC BY). http://creativecommons.org/licenses/by/4.0/

(c) (i) Open Access

\begin{abstract}
Tuberculosis remains a public health problem in developing country particularly in Tunisia. Bone location of tuberculosis infection is increasing and is often misdiagnosed due to the weakness of clinical presentation in early stages. Sternoclavicular joint tuberculosis is rare and unusual location of this disease. However, antibiotherapy and surgical debridement is still the basis of treatment. We report a case of sternoclavicular joint tuberculosis with a follow up of four years. The patient was treated surgically and put under antibiotherapy during twelve months. The site was sterilized. We report this case to show that debridement and antibiotherapy still efficient in tuberculous bone affection no matters the location.
\end{abstract}

\section{Keywords}

Tuberculosis, Sternoclavicular, Joint, Treatment, Outcome

\section{Introduction}

Osteoarticular tuberculosis is increasing and is often misdiagnosed in the initial stages due to lack of awareness, or presentation at uncommon sites. Tuberculosis of bones and joints accounts for $1 \%-3 \%$ of patients with tuberculosis [1].

It represents $10 \%$ to $19 \%$ of the osteoarticular attacks. All the bones can be hit but the affection prevails over the long bones (80\%), the epiphyses are mostly hit (50\%) and the lesion of the great trochanter is most frequent. The beginning of a tubercular osteitis is always achieved inside the bone. Bone necrosis, and pus formation lead more or less quickly to the formation of an abscess which appears on the skin and becomes a fistula .With the resurgence of tuberculosis (TB), even in the developed countries, more infections at unusual sites are being de- 
veloped. Sternoclavicular joint (SCJ) is a rare site for the occurrence of tubercular arthritis. It accounts $1 \%-2 \%$ of all cases of peripheral tubercular arthritis [2]. An extensive literature review found only 26 documented cases of sternoclavicular tubercular arthritis.

We present here a case of tubercular arthritis of the sternoclavicular joint in a 63-year-old healthy patient with a special attention to the difficulty of diagnosis and therapeutic options.

\section{Case Report}

A 63-year-old male laborer presented in the outpatient department with painless swelling over the right sternoclavicular region for the last 3 months. He denied any history of fever, chronic cough, dyspnea, loss of appetite or weight loss. Physical examination showed a well-developed man. Body temperature was $37.3^{\circ} \mathrm{C}$, pulse rate was $84 / \mathrm{min}$, and blood pressure was $120 / 70 \mathrm{mmHg}$. The swelling was globular, cystic; with no pulsate and tender to touch over the right SCJ, measuring $6 \times 8 \mathrm{~cm}$ (Figure 1). The skin was stretched and shiny, but there was no sinus. There was no cervical or axillaries lymphadenopathy. The palpation of the remaining clavicle was not remarkable. The shoulder and the scapulothoracic movements were normal and painless.

Laboratory findings on admission revealed hemoglobin $10.3 \mathrm{~g} / \mathrm{dl}$; total leukocyte count (TLC) of $9.000 / \mathrm{mm}^{3}$, with mild lymphocytosis and an erythrocyte sedimentation rate (ESR) of $50 \mathrm{~mm}$ in the $1^{\text {st }}$ hour (Witrabes method). Mantoux (tuberculin) test was positive with an indurations of $20 \mathrm{~mm}$. Chest radiograph (PA view) revealed a normal lung parenchyma, without remarkable bone destruction in the sternoclavicular joint (Figure 2). Computed tomography of the chest showed a heterogeneously enhanced mass lesion, which expanded over the right sternoclavicular joint and the surrounding tissues with a widening of the joint space and a subchondral sclerosis of the sternum (Figure 3). MRI was not available. Due to the big size of the mass, we opted for an

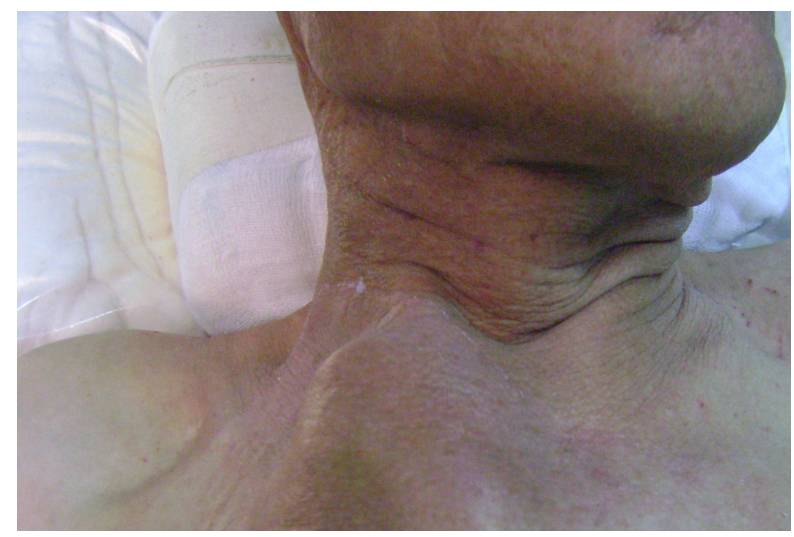

Figure 1. Swelling in front of the right sternoclvicular joint.

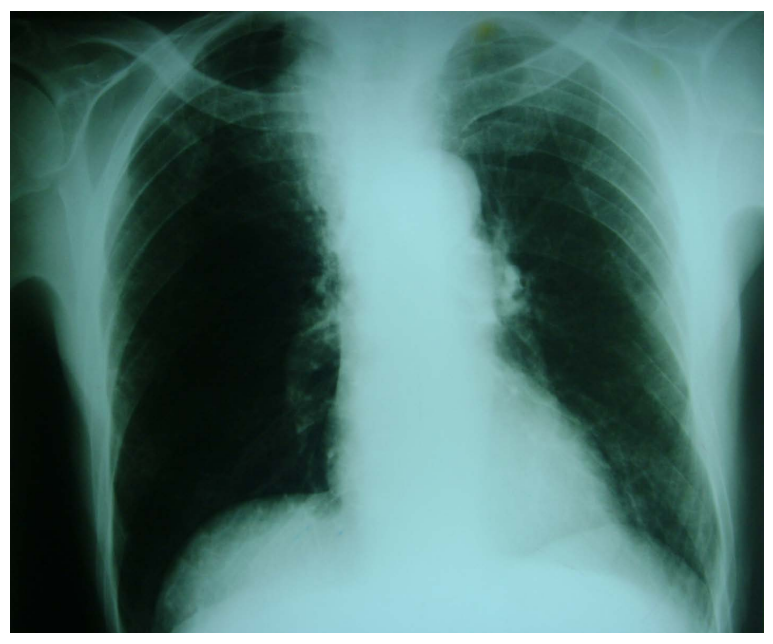

Figure 2. Normal chest X-ray. 


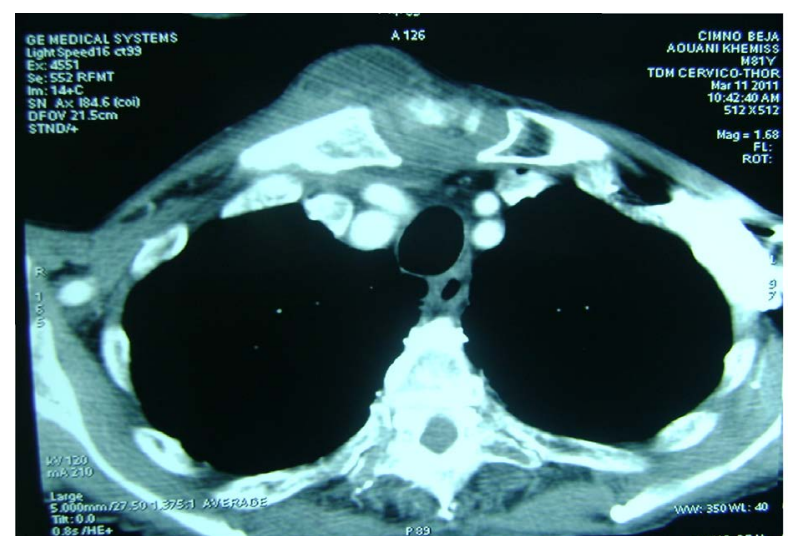

Figure 3. Chest CT-Scan: heterogeneously enhanced mass lesion, which expanded over the right sternoclavicular joint and the surrounding tissues with a widening of the joint space and a subchondral sclerosis of the sternum.

open biopsy and debridement of inflamed tissues. The joint was debrided and washed.

Pathological examination of removed tissue revealed tubercular granulation combining an epithelial and gigantocellular granulation with a caseus necrosis.

Culture for acid fast bacilli was subsequently positive, and Gram's staining or culture did not reveal pyogenic organisms.

The patient was put on a four-drug antitubercular therapy consisting of rifampicin (450 mg daily), isoniazid (300 mg daily), ethambutol (800 mg daily) and pyrazinamide (1500 mg in divided doses) in the initial intensive phase. The four drugs were given for a period of three months followed by rifampicin and isoniazid for nine months. The biopsy wound healed and the swelling subsided in four weeks. At the end of one year, the patient was symptoms-free, with full range of movements. The patient was doing well at the end of a 4-year follow-up.

\section{Discussion}

Due to uncommon nature of osteoarticular TB, presentation at unusual sites like the sternoclavicular joint may not bring this diagnosis to mind. Septic arthritis of the SCJ with staphylococcus aureus and pseudomonas aeroginosa is more common (9\%) than mycobacterial involvement of the joint (1\% - 2\%) [3]. Two of the world's largest series of cases of sternoclavicular TB show an incidence loss than $0.5 \%$ of sternoclavicular involvement [4] [5]. Of the 1074 cases of osteoarticular tuberculosis reported by Tuli SM, only seven cases were reported to be involving the clavicle and the sternoclavicular joint.

In a series of 75 cases of tuberculosis of the upper limb joints, tubercular of the sternoclavicular joint was reported in only one case [4]. In addition to the unusual site of the disease, the usual presentation is an insidious onset of swelling of the joint with minimal pain unlike the rapid onset seen in septic arthritis. Dhillon and al [6] reported series of 9 patients with tuberculous arthritis in 10 sternoclavicular joints. Clinical presentation varied considerably in the 9 patients. The 3 presenting complains in decreasing order of frequency were painful swelling ( 7 joints), painless swelling ( 2 joints) and discharging sinus (1 joint) with a mean duration of symptoms of 13 (6 - 32) months. Pain is not significant in the early phase of the disease; painless swelling may be present for long periods before the appropriate diagnosis is made.

Yasuda et al. reported 3 patients with tubercular arthritis in the SCJ. Painless swelling was the commonest mode of presentation ( $3 / 3$ joints). In our case, pain was neither severe as would be expected in cases having septic arthritis, nor were local symptoms very marked. The swelling was however very palpable. Tuberculosis of the SCJ may be suspected in patients with chronic unexplained shoulder pain. Underlying debilitating disorders, corticosteroids therapy, malnutrition, low socio-economic status, ethanol abuse, history of exposure to tuberculosis, HIV infection and immunocompromised states are known risk factors for tuberculosis. [1] [7]

Due to confluence of structures, this region is poorly visualized by plain radiographs [8]. Because tubercular arthritis of the sternoclavicular joint may cause a retrosternal abscess with or without mediastinitis we believe that modern imaging methods like CT scan and MRI delineates the abscess better in the soft tissues and shows 
joint effusion, which is hyperintense on T2-weighted images. [9] Shah et al. [9] have described the imaging features in 15 cases of sternoclavicular tubercular arthritis. Five of their cases involve the sternum alone and two the clavicle alone without any involvement of the SCJ. Eight patients had SCJ tuberculosis, of whom six revealed destruction in the clavicle and five had sternal involvement. All the patients who undergone MRI had marrow signal density changes in the sternum and clavicle, the marrow appearing hypointense on T1- and hyperintense on T2-weighted images. MRI is the best radiographic technique for the early detection and diagnosis [9]. Financial limitations and lack of MRI availability in most of the hospitals in our country makes the latter a difficult procedure of choice. The pathogenesis and source of infection in all cases of sternoclavicular TB is believed to be fresh or reactivated pulmonary focus [3] which has spread hematologically. However, in the region of the SCJ, contiguous involvement from apical pulmonary lesions has been implicated. Contrary to our findings, Yasuda et al. [8] found evidence of pulmonary TB in all three of their cases, with one case having probably contiguous spread. Martini [4] felt that the primary focus could be the medial end of the clavicle. Differential diagnosis of the SCJ involvement should include fungal and other infections, rheumatoid arthritis, osteoarthrosis and and Tietze syndrome. Other conditions in which swelling near the SCJ mimics tuberculosis infection at this site include sternoclavicular hyperostosis, condensing osteitis, and necrosis with infection [10]. Once necrotic tissue or pus is aspirated, the commonest confusion is with the much more frequent pyogenic infections. It is thus, imperative that some form of tissue diagnosis should be undertaken at an early stage of disease. The tubercle bacillus itself is very difficult to culture and a histological diagnosis of chronic granulomatous infection with some caseus necrosis should warrant a trial of antitubercular drugs. Possible complications include fistula formation, spontaneous fractures of the sternum or clavicle, compression or erosion of the large blood vessels at the base of the neck, compression of the trachea and migration of tubercular abscess into the mediastinum, pleural cavity, subcutaneous tissues or the neck [2] [8]. Secondary infection of these collections can also occur. As Yasuda and Dhillon [6] [8], we believe that in florid infection with abscess formation, combination of surgical debridement and antituberculous drug therapy is essential. Dhillon et al. [6] have described a case report of bilateral SCJ tuberculosis. The left side had to be debrided at a second stage in spite of antituberculous therapy before the disease process resolved. The right side had been debrided at the time of initial biopsy. As same, Yasuda et al. [8] have treated their 3 presented cases with thorough operative debridement and systemic administration of antituberculous agents. They recommend antituberculous agents alone if the infection diagnosed at an earlier stage. Anti tuberculosis chemotherapy should be administered for at least twelve months.

\section{Conclusion}

In summary, we reported a tubercular arthritis involving the sternoclavicular joint and surrounding tissues that mimicked a tumor in an immunocompetent patient. The disease did not recur in our patient, and the results of treatment were good. The key to optimal results is early diagnosis and appropriate treatment. Modern imaging techniques like CT scan and MRI, although expensive, and not routinely available in third world settings, have an important role to play in the diagnosis. Advanced tuberculosis arthritis of the SCJ should be treated with a combination of surgical debridement and anti tuberculosis therapy.

\section{References}

[1] Watts, H.G. and Robert, L. (1996) Tuberculosis of Bones and Joints. Journal of Bone and Joint Surgery, 78, $288-299$.

[2] Bezza, A., Niamane, R., Benbouazza, K., et al. (1998) Tuberculosis of the Sternoclavicular Joint: Report of Two Cases. Revue du Rhumatisme English Edition, 65, Article ID: 7916794.

[3] Enarson, D.A., Fuji, M., Nakielna, E.M. and Grzybowsky, S. (1979) Bone and Joint Tuberculosis: A Continuing Problem. Canadian Medical Association Journal, 120, 139-145.

[4] Martini, M. (1988) Tuberculosis of the Bones and Joints. Springer-Verlag, Berlin, 149-150. http://dx.doi.org/10.1007/978-3-642-61358-6_12

[5] Tuli, S.M. (1993) Tuberculosis of the Skeletal System. Jay Pee Brothers Medical Publisher, New Delhi, 121-123.

[6] Dhillon, M.S., Gupta, R., Rao, K.S. and Nagi, O.N. (2000) Bilateral Sternoclavicular Joint Tuberculosis. Archives of Orthopaedic and Trauma Surgery, 120, 363-365. http://dx.doi.org/10.1007/s004020050486

[7] Fukasawa, H., Suzuki, H., Kato, A., Yamamoto, T., Fuigaki, Y. and Yonemura, K. (2001) Tuberculous Arthritis Mimicking Neoplasm in a Hemodialysis Patient. The American Journal of the Medical Sciences, 322. http://dx.doi.org/10.1097/00000441-200112000-00012 
[8] Yasuda, T., Trauma, K. and Fujiwara, M. (1995) Tuberculosis Arthritis of the Sternoclavicular Joint—A Report of Three Cases. Journal of Bone and Joint Surgery, 77,136-139.

[9] Shah, J., Patkar, D., Parikh, B., Parmar, H., Varma, R., Patankar, T. and Prasad, S. (2000) Tuberculosis of the Sternum and Clavicle: Imaging Findings in 15 Patients. Skeletal Radiology, 29, 447-453.

http://dx.doi.org/10.1007/s002560000207

[10] Yood, R.A. and Goldenberg, D.L. (1980) Sternoclavicular Joint Arthritis. Arthritis and Rheumatism, 2, 232-239. 